\title{
75 COMPREHENSIVE PROFILING OF THE TUMOR-IMMUNE MICROENVIRONMENT USING AN AUGMENTED TRANSCRIPTOME
}

Eric Levy*, Pamela Milani, Fabio Navarro, Gabor Bartha, Charles Abbott, Jose Jacob, Rena McClory, Robin Li, John West, John Lyle, Sean Boyle, Richard Chen. Personalis, Inc., Menlo Park, CA, USA

Background Comprehensive profiling of both the tumor and tumor microenvironment (TME) can help further our understanding of tumor progression and response to treatment. Many immune features can be extracted from transcriptomic data, including characterization of the immune infiltrate and profiling the diversity of immune receptors. To address this, we have developed multiple TME profiling features as part of the ImmunoID NeXT Platform ${ }^{\circledR}$, an augmented, immunooncology-optimized exome/transcriptome platform designed to provide comprehensive information regarding the tumor and TME from a single FFPE tumor sample. These features including quantification of immune cell infiltration and profiling of the T-cell receptor (TCR) and B-cell receptor (BCR).

Methods To develop our immune infiltrate quantification method, we profiled the transcriptomes of eight purified immune cell types using ImmunoID $\mathrm{NeXT}^{\mathrm{TM}}$ to develop platform-specific gene sets, and compared our transcriptome quantification to immune cell quantification with IHC. For TCR and BCR methods, we analyzed the reproducibility of clone results, and compared top clones to standalone TCR and BCR sequencing approaches. In addition, we characterized the immune content of over 800 tumor samples across 14 cancer types. Finally, we analyzed the immune features in a cohort of melanoma patients who underwent PD-1 blockade.

Results We observe significant concordances between cell fractions by IHC and ImmunoID NeXT's transcriptome-based scores in tumor FFPE samples for B cells, CD8 + T cells, and macrophages (R2>0.82, R2 $>0.75$, and $\mathrm{R} 2>0.52$, respectively). For TCR and BCR methods, abundances of clones shared between subsequent curls of a tumor FFPE sample have very high concordances (R2>0.89, R2 $>0.92$, and $\mathrm{R} 2>0.76$ for TRB, IgG, and IgA, respectively). Compared to the standalone approaches, we identify $100 \%$ of the top 500 TRB clones and $95 \%$ of the top $500 \mathrm{IgG}$ clones, with highly concordant abundances (R2 $>0.94$ and $\mathrm{R} 2>0.82$ for TRB and IgG, respectively) in a PBMC sample. We identify biologically-relevant immune signatures across tumor types by characterizing the immune features across over 800 tumor samples. Finally, in a melanoma cohort, TRB clonality and CD8 $+\mathrm{T}$ cell scores are significantly different in responders to checkpoint inhibition.

Conclusions RNA sequencing can be used as a scalable approach to profile the immune composition in tumors. Such analysis can add to our understanding of the tumor-immune interaction, including studies of response to immunotherapy. We show that immune infiltrate quantification and TCR and BCR profiling - all part of the ImmunoID NeXT Platform are able to accurately and effectively evaluate the composition and diversity of tumor-infiltrating immune cells.

http://dx.doi.org/10.1136/jitc-2021-SITC2021.075 